\title{
Le gisement acheuléen de La Grande Vallée à Colombiers (Vienne, France) : stratigraphie, processus de formation, datations préliminaires et industries lithiques
}

David Hérisson, Jean Airvaux, Arnaud Lenoble, Daniel Richter, Émilie Claud et Jérôme Primault

\section{(2) OpenEdition}

\section{Journals}

Édition électronique

URL : http://journals.openedition.org/paleo/2329

DOI : $10.4000 /$ paleo.2329

ISSN : 2101-0420

Éditeur

SAMRA

Édition imprimée

Date de publication : 15 décembre 2012

Pagination : 137-154

ISSN : $1145-3370$

Référence électronique

David Hérisson, Jean Airvaux, Arnaud Lenoble, Daniel Richter, Émilie Claud et Jérôme Primault, "Le gisement acheuléen de La Grande Vallée à Colombiers (Vienne, France) : stratigraphie, processus de formation, datations préliminaires et industries lithiques », PALEO [En ligne], 23 | 2012, mis en ligne le 19 avril 2013, consulté le 24 juillet 2020. URL : http://journals.openedition.org/paleo/2329 ; DOI https://doi.org/10.4000/paleo.2329

Ce document a été généré automatiquement le 24 juillet 2020

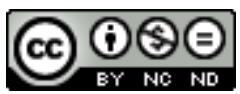

PALEO est mis à disposition selon les termes de la licence Creative Commons Attribution - Pas d'Utilisation Commerciale - Pas de Modification 4.0 International. 


\section{Le gisement acheuléen de La Grande Vallée à Colombiers (Vienne, France) : stratigraphie, processus de formation, datations préliminaires et industries lithiques}

David Hérisson, Jean Airvaux, Arnaud Lenoble, Daniel Richter, Émilie Claud et Jérôme Primault

\section{Introduction}

1 Depuis une trentaine d'années, les recherches sur les dépôts du début du Pleistocène moyen se sont multipliées en Europe occidentale. C'est, entre autres, le cas en Angleterre avec Boxgrove (Roberts et al. 1997) et Happisburgh (Parfitt et al. 2010), dans le nord de la France avec Cagny (Tuffreau et al. 2008), Menez-Dregan (Hallegouët et al. 1992) et Soucy (Lhomme 2007), le Centre de la France avec La Noira (Despriée et al. 2010), en Italie avec Notarchirico (Piperno 1999) et en Espagne avec Atapuerca (Carbonell et al. 2001) et Aridos (Santonja et al. 1980).

2 En Poitou-Charentes, de très nombreux sites acheuléens ont été découverts depuis les débuts de la recherche préhistorique au XIXè siècle. En Charentes, les sablières de la région de Saint-Amand-de-Graves, de Saint-Même-les-Carrières, jusqu'à Jarnac sont bien connues pour avoir livré des industries anciennes (Guillien 1941 ; Patte 1956, 1972 ; Airvaux 1983). Les alluvions de la Charente en Charente-Maritime sont également très riches en vestiges acheuléens. Malheureusement, le système de terrasses est imbriqué ou peu différencié et souvent à la limite de la nappe aquifère, rendant très difficiles les observations. Dans le nord des Deux-Sèvres, en bordure du Massif armoricain, les 
alluvions de La Dive et du Thouet contiennent des industries acheuléennes (Germond 1982). Dans la Vienne, au nord de Châtellerault, plusieurs sites acheuléens sont connus des prospecteurs de surface depuis le XIXéme siècle (Patte 1941). A la Roche-Posay, au nord-est de la Vienne, les alluvions de la Creuse se répartissent entre le département de la Vienne en rive gauche et l'Indre-et-Loire en rive droite. Au lieu-dit La Revaudière, sur la commune d'Yzeures-sur-Creuse, la terrasse de 15-22 m a livré une industrie acheuléenne exceptionnelle (Fritsch 1972; Gratier et Macaire 1981). Ce tour d'horizon de la région Poitou-Charentes met en évidence un contexte riche en découvertes anciennes de sites ayant livré des pièces lithiques attribuées à l'Acheuléen. Cependant, aucun des gisements connus n'a jusqu'à présent fait l'objet de fouille. Le mobilier lithique a été recueilli sans contexte stratigraphique et il n'existe aucun élément de datation directe de ces industries.

Le site de La Grande Vallée, à Colombiers près de Châtellerault, objet du présent article, vient compléter de façon primordiale cet ensemble de découvertes. Les fouilles que nous y avons menées, de 2005 à 2008, apportent une connaissance très complète d'industries acheuléennes issues d'une séquence datée comportant cinq niveaux archéologiques. Les lignes qui suivent posent les premières bases du cadre géologique, stratigraphique et chronologique de ce gisement. Puis, sont décrites et discutées la nature ainsi que les caractéristiques des assemblages lithiques mis au jour.

\section{1 - Contexte géologique, géographique et découverte du gisement}

Aux confins du Bassin parisien et du Bassin aquitain, dans le centre-ouest de la France, la région du Poitou, constitue un seuil géologique (le Seuil du Poitou) prolongeant les extensions respectives du Massif central et du Massif armoricain (fig. 1). Le substrat jurassique affleure en bordure des massifs primaires, enserrant les terrains crétacés caractéristiques des deux grands bassins sédimentaires. Jusqu'en sud-Touraine, l'étage du Turonien supérieur (et moyen parfois) a été fortement altéré. Les altérites argileuses issues de cette transformation comportent une impressionnante quantité de silex tabulaire. C'est dans ce contexte, en bordure des altérites du Turonien supérieur, que se situe le gisement de La Grande Vallée.

Le site se trouve au sud de la Loire, dans le département de la Vienne, à Colombiers près de Châtellerault (fig. 1 et 2). Cette commune occupe le versant septentrional d'un important plateau boisé d'environ $8 \mathrm{~km}^{2}$, qui domine de $90 \mathrm{~m}$ une vallée de plus de 6 $\mathrm{km}$ de largeur au nord, où coule l'Envigne, modeste affluent de la rivière Vienne à Châtellerault (fig. 2).

6 A mi-chemin entre la commune de Colombiers et celle, voisine, de Marigny-Brizay débouche, au lieu-dit La Grande Vallée, un petit talweg court et profond, dont l'axe est d'orientation générale nord. En ce lieu, la petite route reliant Beaumont à Colombiers épouse la courbure du versant ouest du talweg et, notamment celle d'un replat structural qui, $60 \mathrm{~m}$ au-dessus du niveau de base local, se développe à la faveur d'une faiblesse lithologique soulignant le contact entre les assises calcaires du Turonien inférieur et les bancs gréseux du Turonien moyen (fig. 2).

7 En 1995, les travaux de réfection du talus et du fossé de la route ont révélé la présence de dépôts fossiles sur ce replat. De très nombreux artefacts dont des bifaces y ont été 
découverts par l'un d'entre nous (J. Airvaux), alors que la coupe du talus faisait déjà apparaître la présence d'un site important. En 2005, un sondage de $2 \mathrm{~m}^{2}$ situé à environ $1,5 \mathrm{~m}$ en arrière de la coupe du talus de la route fut réalisé. De 2006 à 2008, une opération programmée a permis de fouiller les niveaux archéologiques en place sur 18 $\mathrm{m}^{2}$ et d'étudier la séquence stratigraphique du gisement.

Figure 1- Localisation du gisement de La Grande Vallée dans son contexte géologique régional.

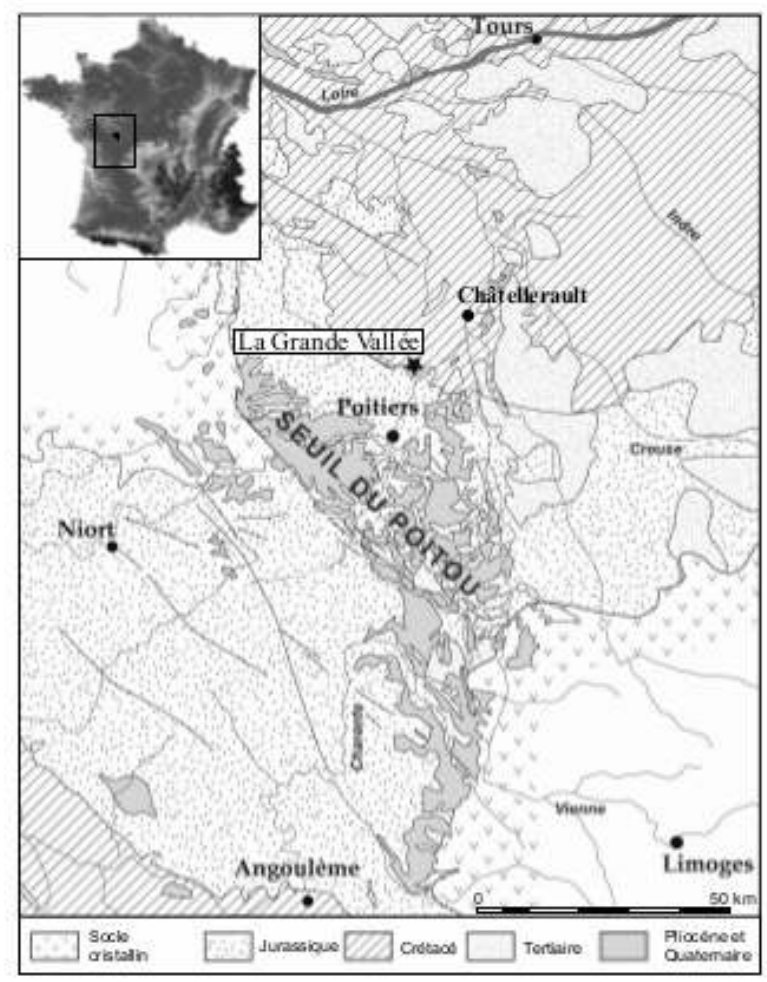

Figure 2 - Localisation du gisement de La Grande Vallée dans son contexte géologique local (extrait de la carte géologique de Bourgueil et al. 1976, modifié).

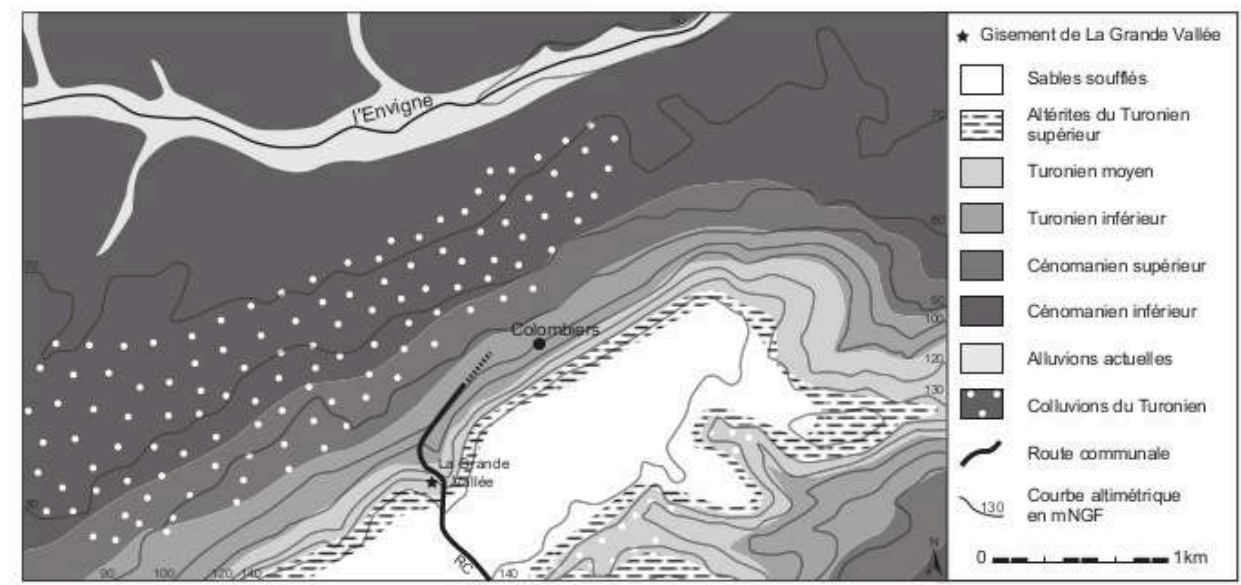




\section{2 - Stratigraphie des dépôts et dynamique sédimentaire}

\section{1 - Description des unités lithostratigraphiques}

Une stratigraphie de trois mètres a été révélée par la fouille, sans que la base des dépôts pléistocènes n'ait été atteinte en l'état actuel des travaux (fig. 3). A partir de l'étude des faciès et des structures sédimentaires, cinq unités ont été distinguées et décrites (tabl. 1). Les dépôts de base sont formés de 1,4 $\mathrm{m}$ d'argiles sableuses massives brunes panachées à lits sableux intercalés passant à un diamicton riche en cailloux et blocs à l'aval (unité 5). Ils sont surmontés d'une nappe épaisse de 0,6 $\mathrm{m}$ de sables grossiers à granules et cailloux présentant un litage mal exprimé (unité 4), elle-même couverte d'un mince lit sableux (unité 3) puis d'un dépôt semi-métrique d'argiles grossièrement stratifiées par superposition de lits plus ou moins riches en cailloux de toute taille et d'orientation quelconque (unité 2). Un dépôt peu épais de colluvions se superpose de façon non-conforme aux sédiments sous-jacents (unité 1). Outre la fraction archéologique, les éléments grossiers regroupent des débris calcaires silicifiés, des dalles et des gélifracts de silex issus des altérites qui coiffent la butte et des pédoreliques héritées de vieux sols représentées essentiellement par des concrétions d'oxydes de fer. Ces dernières sont principalement localisées dans les unités de la partie supérieure de la séquence.

\section{2 - Dynamiques sédimentaires et phénomènes périglaciaires}


Figure 3 - Log stratigraphique du gisement de La Grande Vallée : unités lithostratigraphique et niveaux archéologiques (les triangles inclinés représentent des ensembles archéologiques non homogènes provenant d'une accumulation issue de l'érosion de nombreuses occupations du versant).

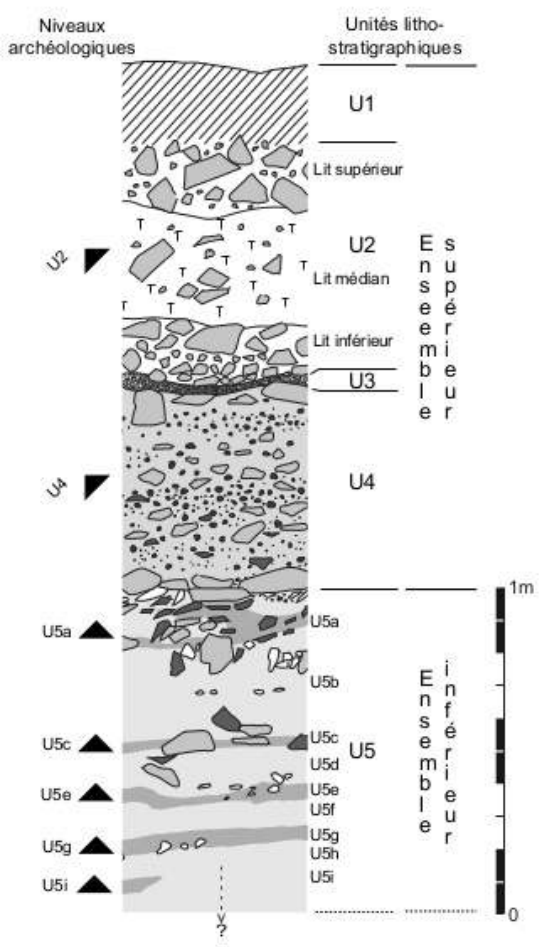

Tableau 1 - Description des unités lithostratigraphiques. La description des fabriques des dépôts suit la terminologie proposée par Benn (1994).

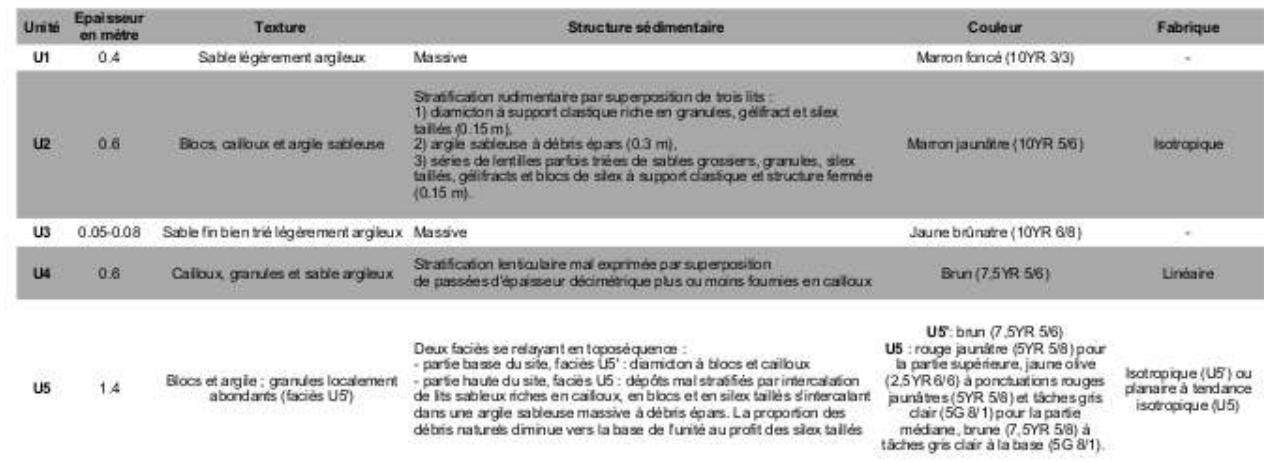


Figure 4 - $A$ - vue générale du niveau $5 \mathrm{~g}$ décapé ; $B$ - vue rasante du niveau $5 \mathrm{~g}$ décapé ; $\mathrm{C}$ - vue partielle du niveau 5 e décapé, le cadre blanc en pointillé correspond à la zone couverte par la vue D ; D - vue rasante du niveau 5e décapé. Clichés D. Hérisson
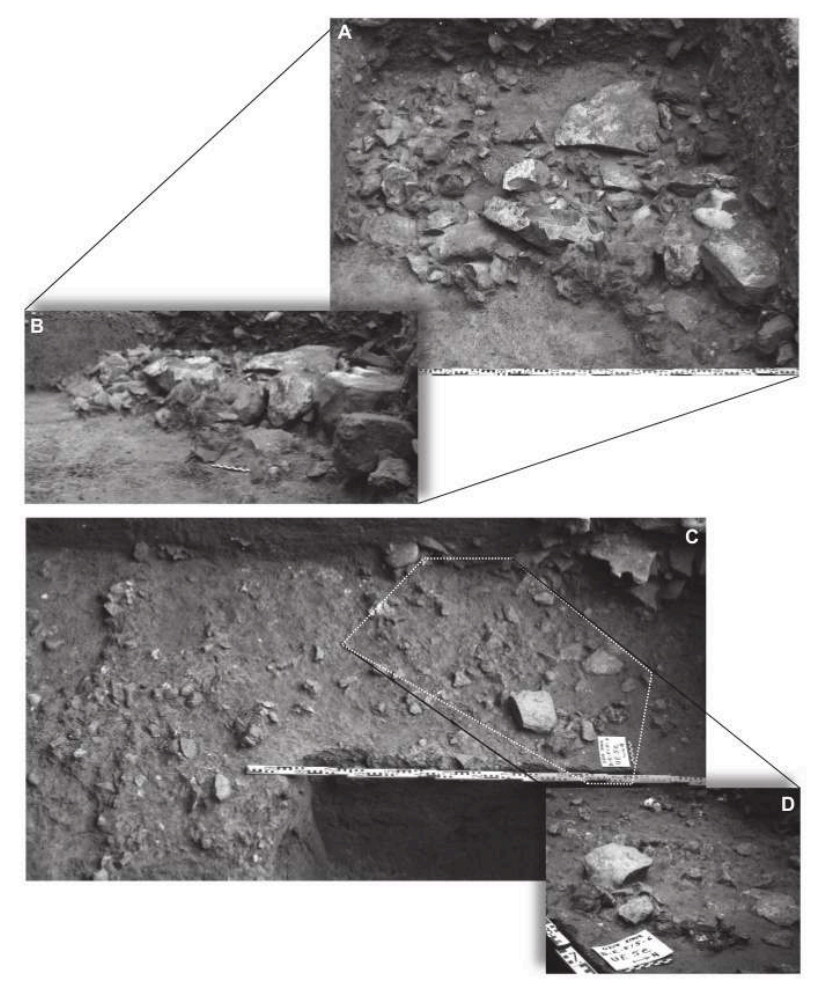

9 La fabrique isotrope, le support matriciel, le mauvais tri des cailloux et le litage mal exprimé de l'unité 2 désignent un dépôt édifié par des coulées de débris successives (Bertran et Texier 1999). L'excellent tri des sables et les figures de tri observées sous le microscope indiquent que l'unité 3 est un dépôt de ruissellement (Lenoble 2005). Ces mêmes critères et l'abondance de fraction fine montrent que ce processus a également contribué à l'édification des dépôts de base (unité 5). Mais, dans cette dernière unité, le ruissellement ne tient qu'un rôle secondaire par comparaison à la solifluxion. Le terme de solifluxion est utilisé ici dans le sens de solifluxion périglaciaire (Washburn 1979; Harris et al. 1997). Il désigne le lent déplacement d'un sol soumis à des cycles gel-dégel sur des pentes supérieures à $2^{\circ}$ ou $3^{\circ}$. Ce déplacement, de l'ordre de un à quelques centimètres par an, l'action de différents processus, à savoir le gonflement du sol lors de l'engel à la suite de la formation de glace de ségrégation dans les sédiments, son affaissement au dégel, le déplacement individuel des artefacts en surface par suite de la formation d'aiguilles de glace et des épisodes de liquéfaction des sédiments au dégel. Sa contribution à la sédimentation est attestée par les microstructures cryogéniques des dépôts (structure lamellaire ou granulaire, Murton et French 1994), les déformations des lits sableux (étirement et boudinage) et les morphologies en lobes mises en évidence par la fouille (fig. 4). Ces lobes ont été observés et relevés dans les zones de transition entre le faciès argileux de la zone amont et l'accumulation de blocs de la zone aval. Ils sont formés de concentrations de cailloux et de dalles de silex, allongées et arquées, s'étendant sur un à deux mètres et disposées transversalement à la pente (fig. 4). De tels bourrelets sont observés dans les milieux périglaciaires actuels semidésertiques soumis à la solifluxion. Les cailloux formant les pavages au dos des coulées se déplacent d'autant plus rapidement qu'ils sont gros; ils constituent des 
concentrations aux fronts des coulées. Une telle organisation est caractéristique de la solifluxion à front pierreux qui se développe généralement sur des pentes faibles à moyennes (Bertran et al. 1995). L'empilement de ces fronts est à l'origine du faciès de blocs et de cailloux observé à l'aval de l'emprise de fouille. Ce dernier correspond donc à une zone d'immobilisation des coulées en pied de versant. Le tri de la fraction grossière, les granoclassements inverses et l'orientation préférentielle conforme à la direction de pente des éléments allongés désignent également l'unité 4 comme un dépôt de solifluxion périglaciaire. Le faciès n'est cependant pas celui d'un empilement de lobes, mais celui d'une superposition de coulées par ailleurs fortement lessivées (absence de fraction fine, figures de lessivages observées sous le microscope : plages lavées, coiffes silteuses). Ces caractéristiques sont celles d'un dépôt formé dans la zone de transit.

La succession des différents termes de la séquence traduit donc une aggradation et une régularisation $\mathrm{du}$ versant. Les premiers dépôts observés sont des accumulations distales (unité 5), surmontés par des dépôts de la partie médiane du versant (unité 4 et 3) auxquels succède une sédimentation discontinue par superposition de coulées de débris (unité 2). La discordance angulaire qui sépare cette dernière unité des précédents dépôts montre par ailleurs que ces épisodes des coulées de débris accompagnent l'accroissement d'un cône colluvial venant se superposer au versant précédemment régularisé.

\section{3 - Premiers éléments de datation du gisement}

\section{1 - Chronostratigraphie et estimation de l'âge des industries}

11 La durée de formation de la séquence peut être estimée sur la base des observations paléopédologiques relevées sous le microscope. Ces dernières se regroupent en trois catégories : des traits hydromorphes (plages dégradées ou plages imprégnées d'oxydes), des traits cryogéniques (structure lamellaire ou granulaire, figures de lessivage) et des revêtements d'argiles microlitées en relation avec le développement de luvisols (Jamagne 2008). L'épaisseur des accumulations d'argiles et la puissance des horizons où elles se distribuent excluent des sols formés à la faveur d'amélioration climatique de type interstade. Les sols lessivés auxquels se rattachent ces horizons argiliques sont donc de rang interglaciaire. Trois horizons liés à trois luvisols fossiles ont été identifiés. Entre ceux-ci s'intercalent des sols structurés par le gel, en particulier à la base des dépôts où la solifluxion est l'agent principal de sédimentation. Les traits hydromorphes observés sont ubiquistes et peuvent se rattacher aux différents types de sols distingués. La surimposition d'un horizon argilique à un profil structuré par le gel (horizon granulaire surmontant un horizon à structure lamellaire) forme ainsi le bilan d'un cycle glaciaire - interglaciaire. Ces bilans peu détaillés reflètent un site à faible accrétion, formé sur un temps long - trois cycles glaciaires-interglaciaires distincts étant enregistrés dans la séquence. L'horizon argilique fossile le plus récent est observé en sommet de séquence où il est tronqué par les dépôts les plus sommitaux (unité 1). Le caractère non-conforme des dépôts de cette dernière unité, leur nature colluviale et le sol jeune qu'il supporte montrent que cette unité sommitale est formée de sédiments en transit dans le segment amont du versant en cours d'érosion. On voit ainsi dans cette unité le témoignage d'une évolution du versant en réponse au creusement du vallon «La Grande Vallée » et à l'abaissement subséquent du niveau de base. Cette 
évolution du niveau de base implique un temps long de morphogenèse qui exclut de rattacher l'horizon argilique sous-jacent à l'Holocène. Pour cette raison, cet horizon pédologique représente a minima un sol formé au cours du stade isotopique 5 , les horizons argiliques sous-jacents pouvant être rattachés respectivement aux stades 7 et 9. L'industrie recueillie à la base de l'unité 5 , contenue dans des dépôts de solifluxion, ne peut être plus jeune que le cycle glaciaire précédent, soit le stade isotopique 10. Un âge de $350 \mathrm{ka}$ est alors une bonne estimation de l'ancienneté de ces industries. Le caractère discontinu de la sédimentation, en particulier de la partie supérieure de la stratigraphie, rend probable la présence de hiatus. Aussi, l'âge des dépôts déduits de la succession des paléosols est-il un âge minimal.

L'ancienneté maximale du gisement peut, quant à elle, être estimée par la prise en compte du taux régional d'incision des vallées. La datation des formations alluviales du cours moyen de la proche vallée de la Creuse témoigne ainsi d'un enfoncement du niveau de base de $10 \mathrm{~cm}$ par millénaire (Voinchet et al. 2010). L'absence locale d'activité tectonique autorise à prendre ce taux en compte et la position du site par rapport au niveau de base local pour estimer l'âge de façonnement du replat. Ce dernier ne saurait ainsi être plus ancien que $600 \mathrm{ka}$. L'estimation des âges minimal et maximal permet ainsi de placer les industries de l'unité 5 dans une fourchette de 350 à $600 \mathrm{ka}$, soit dans le second tiers du Pléistocène moyen.

\section{2 - Premiers éléments de datation des niveaux $5 a$ et $5 c$ par thermoluminescence}

La datation par thermoluminescence (TL) des silex brûlés établit le temps écoulé depuis la dernière chauffe de l'échantillon dans un foyer préhistorique. Cette méthode permet de dater directement une activité humaine qui est réellement visible dans l'enregistrement archéologique. La datation TL utilise l'omniprésence de radiation ionisante qui résulte des états d'excitation des électrons (charges). Ceux-ci sont mesurés par thermoluminescence et procurent la dose totale accumulée (paléodose), qui est divisée par le débit de dose pour donner un âge estimé. Le débit de dose interne est déterminé précisément par activation neutronique (U, Th, K) de l'échantillon, mais la dose gamma externe ne peut être mesurée pour la position exacte des échantillons (des silex brûlés analysés) parce qu'ils sont enlevés au cours de la fouille. Les mesures de la dose gamma sont donc effectuées à partir de dosimètres $\alpha$ 開-Al2O3 :C en plusieurs positions dans les sédiments (tabl. 2) afin de donner un débit de dose gamma externe moyen pour chaque unité stratigraphique sédimentaire du site. Bien que les sédiments aient été classés comme "lumpy» (Schwarcz 1994) à cause de la présence de très gros blocs, les doses gamma externes mesurées sont plutôt homogènes. Ce fait contraste avec la grande variabilité des paléodoses. Le manque de connaissance concernant la dose gamma externe pour chaque échantillon individuellement mène donc à un large spectre des résultats d'âge et entraîne l'utilisation inévitable d'une valeur moyenne. Un âge moyen près de l'âge réel peut être calculé seulement si plusieurs échantillons sont datés (Richter et al. 2010). Le protocole suivi ici a été détaillé dans Richter et al. (ibid.et supplément). Les résultats préliminaires de l'analyse de trois silex brûlés du niveau $5 \mathrm{a}$ sont présentés ci-dessous ainsi que ceux de trois autres du niveau $5 c$ (tabl. 2 et 3 , fig. 5 et 6). Les mesures au spectromètre gamma HPGe n'ont révélé aucun déséquilibre dans la série d'uranium des composants de la fraction fine des sédiments. Ces mesures 
indiquent l'absence de récent déséquilibre et nous assure donc la stabilité du débit de dose gamma externe depuis l'enfouissement des pièces.

La présence de très grandes pièces en silex (à faible concentration en éléments radioactifs) et d'argile comme matrice sédimentaire (à forte concentration en éléments radioactifs) provoque au final un important débit de dose gamma externe. Certains échantillons ont pu se trouver à proximité voire coincés entre des blocs de silex recevant peu de radioactivité tandis que d'autres ont reçu de grandes doses étant majoritairement entourés d'argile.

Les âges préliminaires apparaissent divisés en deux groupes l'un autour de $450 \mathrm{ka}$, l'autre autour de $650 \mathrm{ka}$, avec pour le dernier groupe un échantillon dans chaque niveau (tabl. 4). A cause du faible nombre d'échantillons analysés pour l'instant, on ne peut pas dire s'il s'agit de données aberrantes ou simplement des valeurs extrêmes de la distribution de l'âge. Tous les échantillons ont statistiquement le même âge (2鯆). Cela montre clairement que les deux strates se sont accumulées sur une relative courte période de temps aux environs de $500 \mathrm{ka}$.

Tableau 2 - Débits de dose y externes mesurés avec les dosimètres a Al203 :C.

\begin{tabular}{ccccc}
$\begin{array}{c}\text { Dosimètre } \\
\mathbf{N}^{\circ}\end{array}$ & Niveau & Carré & $\begin{array}{c}\text { Débit de dose } \\
\gamma\left(\mu \mathrm{Gy} \mathbf{a}^{-1}\right)\end{array}$ & $\mathbf{\Xi}$ \\
20 & $5 \mathrm{a}$ & $\mathrm{E} 7 \mathrm{~s} 3$ & 918 & 37 \\
5 & $5 \mathrm{a}$ & $\mathrm{D} 7 \mathrm{~s} 3 / 4$ & 1358 & 267 \\
42 & $5 \mathrm{a}$ & $\mathrm{D} 4 \mathrm{~s} 1 / 2$ & 888 & 74 \\
6 & $5 \mathrm{a}$ & $\mathrm{C} 7 \mathrm{~s} 4$ & 868 & 66 \\
9 & $5 \mathrm{a}$ & $\mathrm{E} 7 \mathrm{~s} 4$ & 979 & 8 \\
11 & $5 \mathrm{a}$ & $\mathrm{E} 4 \mathrm{~s} 2$ & 877 & 62 \\
12 & $5 \mathrm{a}$ & $\mathrm{E} 4 \mathrm{~s} 1$ & 852 & 48 \\
47 & $5 \mathrm{a}$ & $\mathrm{B} 7 / \mathrm{B} 8$ & 987 & 64 \\
113 & $5 \mathrm{a}$ & $\mathrm{C} 9 \mathrm{~s} 4$ & 1010 & 35 \\
137 & $5 \mathrm{a}$ & $\mathrm{B} 7 \mathrm{~s} 4$ & 825 & 69 \\
34 & $5 \mathrm{a}$ & $\mathrm{B} 8 \mathrm{~s} 2$ & 900 & 38 \\
\hline Moyenne & $5 \mathrm{a}$ & & 951 & $\mathbf{1 4 7}$ \\
17 & $5 \mathrm{c}$ & $\mathrm{F} 7 \mathrm{~s} 3$ & 559 & 27 \\
39 & $5 \mathrm{c}$ & $\mathrm{E} 7 \mathrm{~s} 3$ & 1075 & 68 \\
53 & $5 \mathrm{c}$ & $\mathrm{E} 4 \mathrm{~s} 2$ & 1048 & 84 \\
24 & $5 \mathrm{c}$ & $\mathrm{D} 7 \mathrm{~s} 3 / 4$ & 1175 & 43 \\
23 & $5 \mathrm{c}$ & $\mathrm{E} 4 \mathrm{~s} 1$ & 924 & 65 \\
57 & $5 \mathrm{c}$ & $\mathrm{D} 4 \mathrm{~s} 1 / 2$ & 937 & 8 \\
\hline Moyenne & $\mathbf{5 c}$ & & 953 & $\mathbf{2 1 4}$
\end{tabular}

Tableau 3 - Valeurs préliminaires des mesures TL, analyse radiochimique (NAA) et débits des doses.

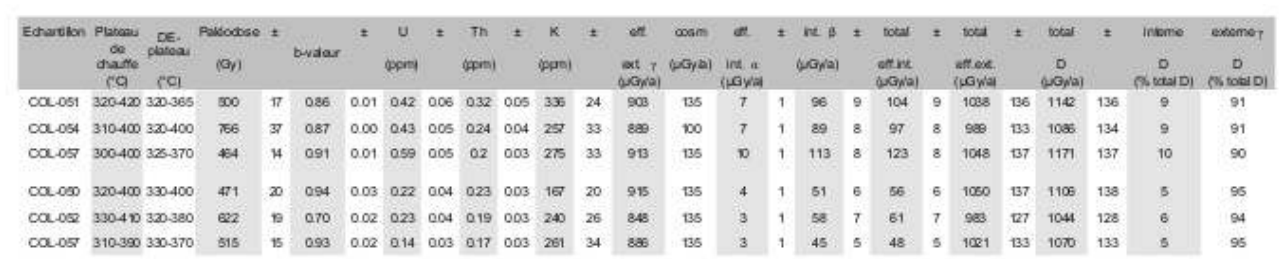




\section{4 - Les industries lithiques}

\section{1 - Taphonomie et implication des processus sédimentaires dans la nature des assemblages lithiques}

De l'industrie lithique est présente dans les unités 2, 4 et 5. Au sommet de la séquence, il ne s'agit que de quelques pièces dispersées. Le nombre d'artefacts augmente progressivement pour former l'essentiel de la fraction sédimentaire grossière à la base des dépôts. Les vestiges contenus dans les unités 2 et 4 sont triés comme le sont les débris naturels. Ces pièces sont manifestement redistribuées, représentant une fraction résiduelle remobilisée à chaque période de sédimentation du versant. Ces multiples phénomènes de remobilisation ont eu pour conséquence une forte dégradation de l'état des pièces qui sont fracturées, possèdent des arêtes très émoussées ou écrasées et parfois un voile blanchâtre de patine. Tel n'est pas le cas des objets contenus dans l'unité 5 , en particulier à sa base, où les vestiges archéologiques représentent la majorité, si ce n'est la totalité de la fraction figurée. L'absence de tri des pièces archéologiques, dans des dépôts de pied de versant, est en accord avec l'hypothèse d'un apport anthropique. Les vestiges n'en sont pas moins redistribués par solifluxion : la concentration des pièces dans les fronts des coulées, l'orientation des éclats allongés transversalement à la pente au sein des fronts et, ailleurs, parallèle à la direction de plus grande pente, ainsi que la diminution de la taille des pièces en s'éloignant des lobes sont autant de caractéristiques qui montrent que les vestiges ont été redistribués par solifluxion (Bertran et al. 1997 ; Lenoble et al. 2009). La transformation des niveaux archéologiques qui en résulte reste cependant difficile à apprécier, dans la mesure où l'intensité des dégradations générées par solifluxion est avant tout liée à la durée de fonctionnement du processus (Texier et al. 1998 ; Lenoble et al. 2007).

Pour ces niveaux de l'unité 5 , l'examen tracéologique des pièces et la cohérence archéologique des séries sont des éléments permettant d'apprécier l'impact de cette dégradation qui, dans tous les cas, ne remet pas en cause l'ancienneté des séries bien calées stratigraphiquement. En l'état, les études archéologiques montrent que ces phénomènes de solifluxion semblent avoir affecté des ensembles qui étaient homogènes à l'origine (avant mobilisation/transformation), correspondant tout au plus à quelques occupations proches dans le temps (à l'échelle du Paléolithique inférieur). A l'œil nu, les pièces présentent un excellent état de préservation. Seule l'étude tracéologique a permis de mettre en évidence la présence de polis plats, souvent intensément développés, d'une patine brillante ainsi que la petite taille des esquillements naturels indiquant que les altérations résultent probablement de fortes pressions maintenues dans le temps que de brefs contacts (comme ceux produits par le piétinement ou la chute de blocs). Cette observation est cohérente avec le processus de solifluxion identifié au cours de l'analyse géoarchéologique décrit ci-avant. Eu égard aux conditions de mise en place des dépôts et de préservation, seuls les niveaux de l'unité 5 présentent un intérêt archéologique de premier ordre. Nos propos se focaliseront logiquement sur la description de ces industries ci-après. 
Figure 5 - Courbes de croissance TL de l'échantillon COL-51 : additive (ligne supérieure) et de régénération (ligne inférieure). La ligne pointillée est la régénération mesurée d'après la décroissance, qui fournit la paléodose.

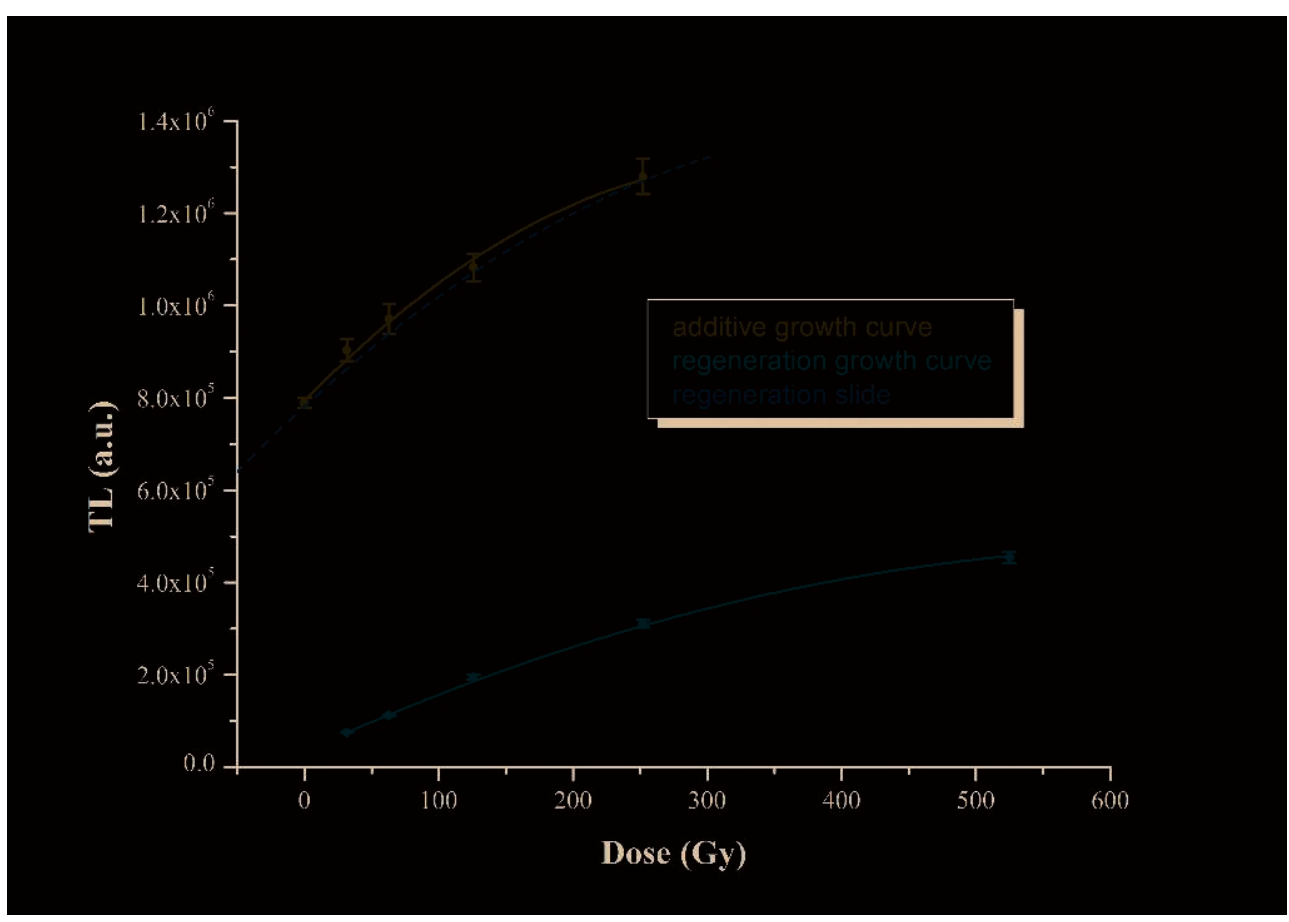

Figure 6 - Courbes de croissance TL naturelle et additive de l'échantillon COL-51 avec le plateau de chauffe en gris.

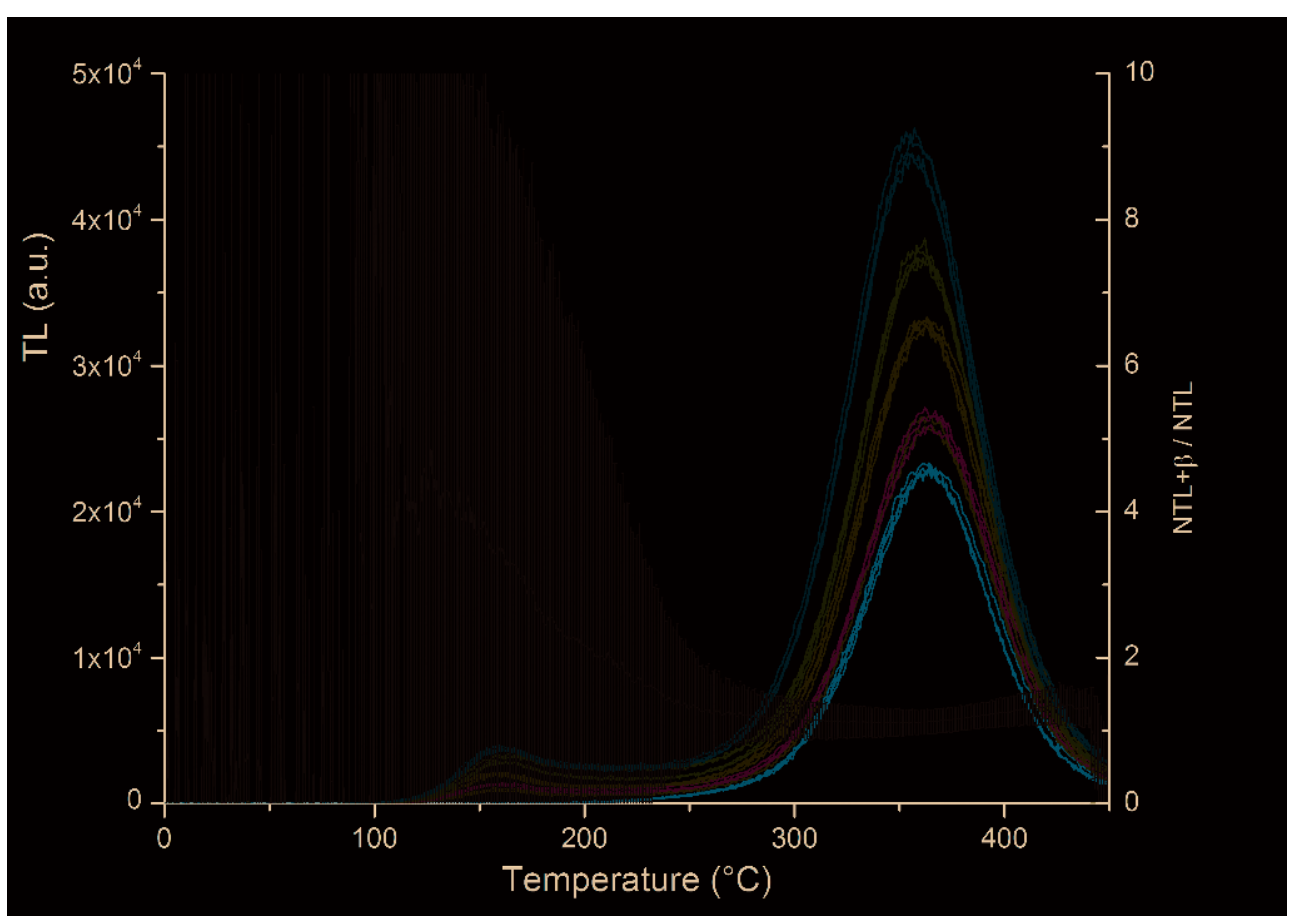


Tableau 4 - Résultats préliminaires des mesures TL et âges préliminaires résultants.

\begin{tabular}{|c|c|c|c|c|c|c|c|}
\hline Echantlion & Niveau & Cane & $\begin{array}{l}\text { Paleodose } \\
\text { (Gy) }\end{array}$ & $\begin{array}{l}\text { Débat de } \\
\text { dose } \\
\text { interne } \\
\text { (uGy a }{ }^{-1} \text { ) }\end{array}$ & $\begin{array}{l}\text { Debit de } \\
\text { dose total } \\
\left(\mu \mathrm{Gy} \mathrm{a}^{-1}\right)\end{array}$ & $\begin{array}{l}\text { Age } \\
\text { (ka) }\end{array}$ & \pm \\
\hline $\mathrm{COL}-51$ & $5 a$ & D $6 s 2$ & 500 & 104 & 1142 & 440 & 60 \\
\hline $\mathrm{COL}-54$ & $5 a$ & $E 5 \& 2$ & 766 & 97 & 1086 & 700 & 90 \\
\hline $\mathrm{COL}-57$ & $5 a$ & D $6 \$ 4$ & 464 & 123 & 1171 & 400 & 50 \\
\hline $\mathrm{COL}-50$ & $5 c$ & E6 $\$ 4$ & 471 & 56 & 1106 & 430 & 60 \\
\hline $\mathrm{COL}-52$ & $5 c$ & $E 6 \& 2$ & 622 & 61 & 1044 & 600 & 80 \\
\hline $\mathrm{COL}-62$ & $5 c$ & E6 s1 & 515 & 48 & 1070 & 480 & 60 \\
\hline
\end{tabular}

\section{2 - Origines de la matière première lithique}

La région de Colombiers constitue l'extension méridionale du Turonien supérieur à grandes dalles siliceuses qui s'étend depuis la région sud-Touraine. Ce silex est surtout connu pour son exploitation au Néolithique récent et final, dans les régions qui s'étendent depuis le nord-est de la Vienne jusqu'au Grand-Pressigny (Indre-et-Loire). La quasi-totalité de la matière première utilisée à La Grande Vallée est ce silex tabulaire du Turonien supérieur. A Colombiers, ce matériau possède parfois des inclusions de macro-bioclastes, en particulier des végétaux (mousses). Cette particularité semble localisée à la région des alentours de Châtellerault. Mis à part ce silex abondamment exploité, d'autres artefacts témoignent de l'utilisation d'autres matières premières lithiques. Quelques silex jurassiques (Bajocien) ont été utilisés par les préhistoriques et pourraient provenir de la région de Poitiers, située à une vingtaine de kilomètres au sud, ou des alluvions du Clain et de la Vienne, rivières proches du site. Un éclat en « jaspe de Fontmaure » a été trouvé et provient de manière certaine de la commune de Vellèches, distante de $30 \mathrm{~km}$ au nord. Il a été également découvert plusieurs objets en "grès lustré » ou quartzite de très bonne qualité d'une splendide couleur pourpre à violette dont le gîte d'origine nous est encore inconnu. Il est possible que cette matière provienne de Fontmaure, mais sans certitude. Un biface a été taillé dans une meulière tertiaire, matière extrêmement abondante dans la moitié est du département et dont la source la plus proche se situe à moins de $10 \mathrm{~km}$ du gisement. En résumé, les Acheuléens de La Grande Vallée se sont déplacés, de façon certaine, dans un rayon minimal de 30 $\mathrm{km}$ autour du site pour s'approvisionner en matière première lithique. La donnée la plus fiable est celle fournie par le «jaspe de Fontmaure » dont le gîte est très localisé. Cependant, comme nous l'avons souligné, la matière première lithique exploitée est très majoritairement locale, disponible en très grande quantité à une petite centaine de mètres en amont de la fouille.

\section{3 - Caractéristiques des industries lithiques de l'unité 5}


Tableau 5 - Décompte général des industries lithiques des niveaux de l'unité 5 à l'issue de la première fouille triennale.

\begin{tabular}{|cccccccccccc|}
\hline Niveau & $\begin{array}{c}\text { Artefacts } \\
\text { non brûlés }\end{array}$ & $\begin{array}{c}\text { Artefacts } \\
\text { brúlés }\end{array}$ & $\begin{array}{c}\text { Total des } \\
\text { artefacts }\end{array}$ & $\begin{array}{c}\text { dont } \\
\text { bifaces }\end{array}$ & $\begin{array}{c}\% \\
\text { biface }\end{array}$ & $\begin{array}{c}\text { dont } \\
\text { ebauches }\end{array}$ & $\begin{array}{c}\text { dont supports } \\
\text { retouchés }\end{array}$ & $\begin{array}{c}\% \text { supports } \\
\text { retouchés }\end{array}$ & $\begin{array}{c}\text { dont } \\
\text { nucléus }\end{array}$ & $\begin{array}{c}\% \\
\text { nucléus }\end{array}$ \\
\hline $5 \mathrm{a}$ & 11464 & 475 & 11939 & 29 & $0,24 \%$ & 10 & 89 & $0,75 \%$ & 14 & $0,12 \%$ \\
$5 \mathrm{c}$ & 2320 & 164 & 2484 & 12 & $0,48 \%$ & 3 & 47 & $1,89 \%$ & 4 & $0,16 \%$ \\
$5 \mathrm{e}$ & 2431 & 251 & 2682 & 12 & $0,45 \%$ & 11 & 35 & $1,30 \%$ & 4 & $0,15 \%$ \\
$5 \mathrm{~g}$ & 1148 & 127 & 1275 & 11 & $0,86 \%$ & 3 & 33 & $2,59 \%$ & 5 & $0,39 \%$ \\
$5 \mathrm{i}$ & 143 & 17 & 160 & 0 & $0,00 \%$ & 0 & 2 & $1,25 \%$ & 1 & $0,63 \%$ \\
\hline Total U5 & 17506 & 1034 & 18540 & 64 & $0,35 \%$ & 27 & 206 & $1,11 \%$ & 28 & $0,15 \%$ \\
\hline
\end{tabular}

Dans le cadre de cet article, nous traiterons les assemblages des différents niveaux de l'unité 5 comme une seule entité dans le but de faire ressortir les caractéristiques essentielles et communes des industries. A l'issue de la première triennale de fouille, 18540 pièces lithiques ont été mises au jour dont 1034 éléments brûlés concernant l'ensemble des niveaux de l'unité 5 (tabl. 5). L'ensemble des étapes des chaînes opératoires est documenté, de l'acquisition de la matière première (cf. §4.2) à l'abandon des outils. Pour les productions très majoritaires en silex du Turonien supérieur, trois types de blocs ont été sélectionnés : des dalles de grandes dimensions (jusqu'à $2 \mathrm{~m}$ pour 8 à $30 \mathrm{~cm}$ d'épaisseur), des petites dalles (d'une trentaine de centimètres pour environ 3 à $7 \mathrm{~cm}$ d'épaisseur) et des rognons ovalaires et plats de dimensions variables.

20 L'objectif principal clairement identifié sur le gisement est la production de bifaces (fig. 7). A cette fin, trois chaînes opératoires ont été mises en œuvre.

21 La première débute par la sélection de dalles de petites dimensions. Ces dernières sont façonnées par une série d'enlèvements alternes ou non au percuteur dur permettant de mettre en forme une zone active. Un dos correspondant au bord de la dalle et une base sont réservés afin de servir de zone préhensible (fig. 7.4). La zone active fait parfois l'objet d'une deuxième étape de façonnage régularisant et affinant l'angle de tranchant par une percussion plus tendre et plus tangentielle que lors de la première phase de façonnage. Le type de biface obtenu possède une forme dissymétrique, la conservation brute de la zone préhensible intégrant le bord de la dalle leur confère un profil caractéristique en coupe en forme de « V ».

22 La deuxième chaîne commence par la sélection de rognons ovalaires de petites et moyennes dimensions. De nombreuses ébauches attestent d'une première mise en forme générale par des séries d'enlèvements au percuteur dur souvent réalisées selon un cheminement dit «de proche en proche». Une seconde phase de finition est effectuée par percussion tendre, organique et tangentielle, comme le prouvent la minceur des éclats de façonnage, leurs très faibles développements conchoïdaux, la morphologie de leurs talons, leur courbure sagittale et la morphologie des négatifs présents sur les bifaces. Certaines pièces présentent une réserve corticale ou zone basale peu investie vouée à la préhension.

23 La troisième chaîne est combinée, mêlant débitage et façonnage (au sens de Brenet 2011). Une première étape consiste à produire des éclats qui seront façonnés dans un second temps. Pour ce faire, les préhistoriques ont sélectionné des rognons ou des plaques de grandes dimensions qu'ils ont fracturés avant débitage. Ces éléments sélectionnés (fragments de plaques ou rognons) ont été débités selon des schémas unipolaires ou centripètes sur une seule face ou par des enlèvements alternes dans un même sens de progression menant parfois au dégagement d'une charnière sur le 
pourtour complet du bloc. Les éclats produits, souvent de grandes dimensions et épais, deviennent le support de la seconde étape de fabrication du biface, le façonnage. Dans certains cas, les éclats font d'abord l'objet d'une phase de mise en forme générale par des séries d'enlèvements au percuteur dur comme pour la deuxième chaîne décrite ciavant. Dans tous les cas, une phase de finition en percussion tendre est mise en œuvre portant un soin particulier au façonnage de la zone active située en partie distale (fig. 7.1, 7.3, 7.5). La majorité des bifaces sur éclat ne possèdent pas de zone proximale non investie mais gardent souvent le « souvenir » de la dissymétrie transverse de leur éclatsupport.

Complémentairement à l'activité d'élaboration des pièces bifaciales, coexiste la fabrication de racloirs et de quelques autres types d'outils plus rares. Ils sont confectionnés à partir d'éclats-déchets issus du façonnage, d'éclats provenant du débitage ou exceptionnellement de cupules de gel récupérées. Les racloirs sont majoritairement simples (fig. 9.3, 9.4, 9.5, 9.8, 9.9). La retouche, souvent très régulière, reste peu envahissante à part pour les supports épais où elle est plus couvrante. Certains racloirs possèdent un amincissement de la région conchoïdale du support. L'outillage est complété par quelques denticulés, encoches de grandes dimensions et par des éléments pouvant être décrits comme des tranchoirs ou hachoirs (fig. 7.6).

Un aspect intéressant du débitage est la recherche de produits allongés, certes en faible nombre. Leur extraction s'effectue à partir des arêtes naturellement anguleuses des bords des dalles menant à l'obtention d'un à trois éclats laminaires (fig. 8.2, 8.3, 8.7). Il n'est pas exclu que ces éclats laminaires aient été utilisés bruts, tout comme les éclats issus du débitage ou les éclats du façonnage.

A ces phases d'acquisition et de production de bifaces et d'outils, particulièrement bien documentées dans le gisement, s'ajoutent celles de l'utilisation et de l'abandon de ces pièces. Ces phases tout aussi fondamentales à explorer sont attestées par la présence d'éclats en "coup de tranchet " ainsi que leurs négatifs sur les bifaces abandonnés. L'étude tracéologique préliminaire apporte des résultats et des éléments-clés pour discuter des modes de fonctionnement de ces pièces avant leur abandon. 
Figure 7 - Productions bifaciales de l'unité 5 de la Grande Vallée : 1, 3, 5 - bifaces ; 2 - éclat coup de tranchet ; 4 - pièce bifaciale à dos ; 6 - grand racloir ou tranchoir. Dessins J.Airvaux.

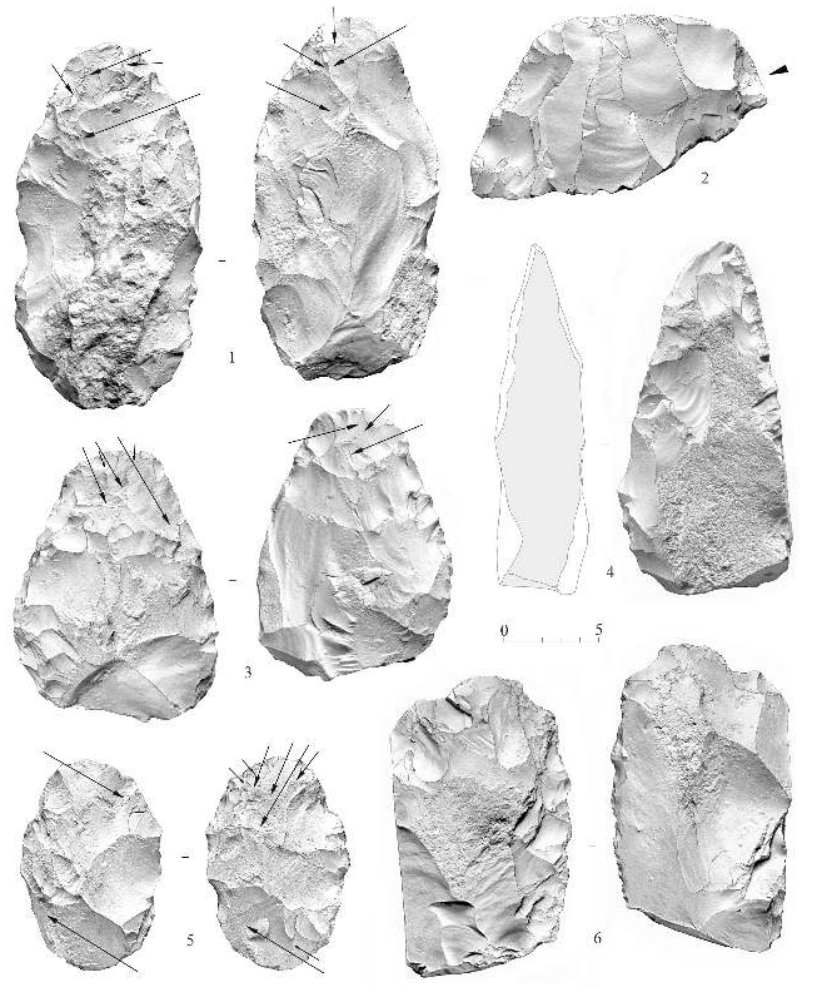

\section{4 - Quelques éléments sur les modes de fonctionnement de l'industrie lithique de l'unité 5}

Après examen à l'œil nu et à la loupe binoculaire de plusieurs centaines de produits recherchés et d'outils retouchés, 52 pièces ont été sélectionnées pour faire l'objet d'analyses tracéologiques, soit parce qu'elles portaient de possibles ou évidentes traces d'utilisation visibles à la loupe binoculaire soit parce qu'elles présentaient un état de conservation correct par rapport au reste de la série. Des éclats bruts, retouchés, des nucléus, un chopping tool et des pièces bifaciales, issus de l'unité 5 (a, c, e et g), composent cet échantillon. La loupe binoculaire (grossissements 10 à 30 fois) et le microscope métallographique (100 à 200 fois) ont été utilisés pour rechercher les différents types de traces d'usures susceptibles d'être rencontrées (esquillements, émoussés, polis, stries) selon la méthode mise en place par S.-A. Semenov (1964) puis développée notamment par R. Tringham et al. (1974), L.-H. Keeley (1977), ou P. Anderson-Gerfaud (1981). Un référentiel expérimental de traces d'utilisation, d'emmanchement, de transport et d'altération (Claud 2008; Claud et al. 2009) a été utilisé afin de comparer les traces archéologiques aux traces expérimentales d'origine connue.

Sur la sélection retenue, 19 pièces portent des traces d'utilisation, sous la forme d'esquillements, d'écrasements et d'arrachements, et un total de 21 zones actives a été enregistré. Le mode de fonctionnement le mieux représenté est la percussion lancée sur des matières dures (fig. 10), son importance étant très probablement la conséquence d'une conservation différentielle des traces. En effet, les altérations, de nature variée (écrasements, émoussés, esquillements, patine brillante, spots brillants et 
stries) et a priori en grande partie d'origine mécanique, sont assez intenses et susceptibles d'avoir détruit les traces les plus ténues. Quelques pièces, néanmoins, portent des esquillements relatifs à la coupe de matières tendres ou tendres à mi-dures. Enfin, des traces d'action mixte (percussion et découpe) sur des matières mi-dures à dures ont également été identifiées.

29 Malgré l'absence de micro-polis d'utilisation, il a été possible, d'après la convergence entre les stigmates mis en évidence et ceux issus du référentiel expérimental, notamment par rapport à la présence ou à l'absence d'écrasements, de distinguer deux grandes catégories de matières (organiques versus minérales) et de proposer pour la plupart des pièces une hypothèse concernant l'activité à laquelle elles se rattachent. Il est ainsi très probable qu'une grande partie des outils $(n=12)$ ait servi dans le cadre de la boucherie, soit pour couper des tissus carnés, soit pour désarticuler en force. Concernant cette activité, les pièces ayant servi en percussion présentent dans leur grande majorité un poids important et une zone préhensive épaisse et confortable opposée à un tranchant non pointu (fig. 10). Ils sont indifféremment bruts ou retouchés en racloirs ou pièces bifaciales. Les outils ayant fonctionné uniquement dans des actions de coupe sont au contraire souvent plus légers et présentent une zone active convergente (deux bifaces et un racloir convergent).

Les traces présentes sur sept pièces restantes (incluant des pièces bifaciales et un nucléus), indiquant un mouvement de percussion lancée contre une matière dure minérale, sont compatibles pour certaines, d'après des expérimentations récemment réalisées (Claud et al. 2010; Thiébaut et al. 2010) avec une utilisation en percuteur de débitage. Ce genre de traces de percussion a d'ailleurs été fréquemment constaté sur les bifaces du Paléolithique inférieur (Wymer 1964; Keeley 1980, 1993 ; Mitchell 1998 ; Wenban-Smith et Bridgland 2001). 
Figure 8 - Eléments de débitage de l'unité 5 de la Grande Vallée : 1 - nucléus unipolaire ; 3 nucléus ayant produit deux éclats laminaires aux dépends de l'arrête du bord de dalle ; 8 - nucléus bipolaire ; 10 - nucléus centripète ; 2,7 - éclats laminaires ; 4, 5, 6, 9 - éclats de débitage. Dessins J. Airvaux.

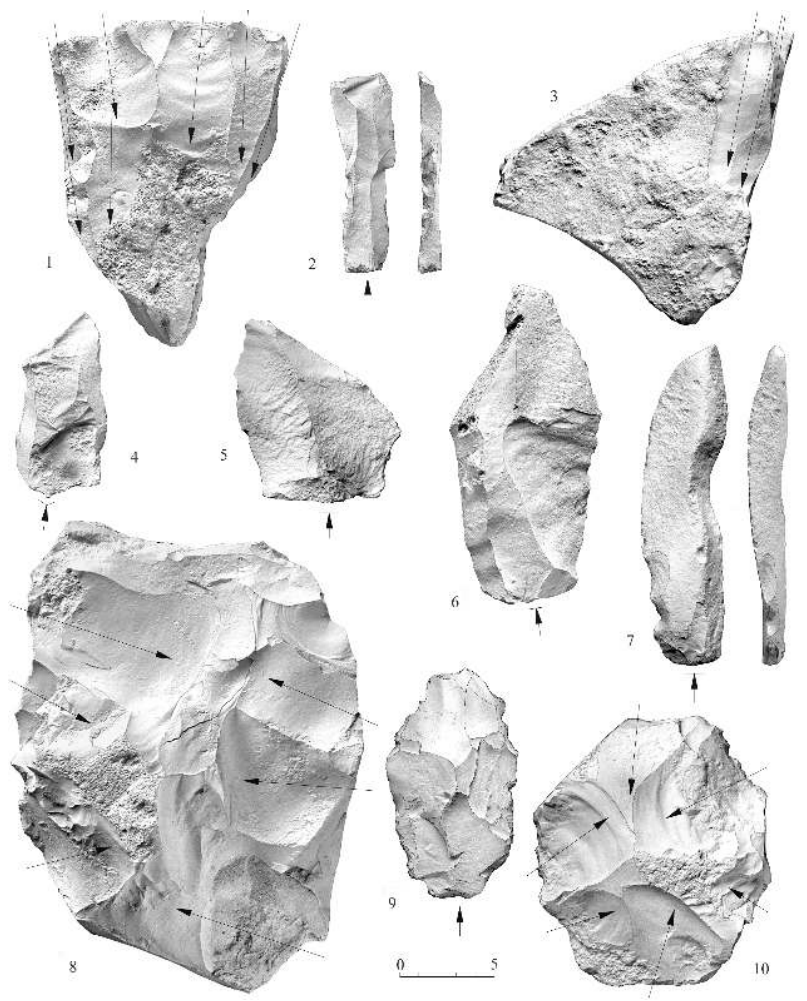


Figure 9 - Supports retouchés de l'unité 5 de La Grande Vallée : 1, 2 - racloirs doubles ; 3, 4, 5, 8, 9 racloirs simples ; $6,7,13$ - racloirs doubles convergents ; 10, 11 - denticulés ; 12 - racloir double convergent avec amincissement en face inférieure ; 14 - pièce à grande encoche ; 15, 16 - grattoir : 17 - éclat retouché de manière liminale. Dessins J. Airvaux.

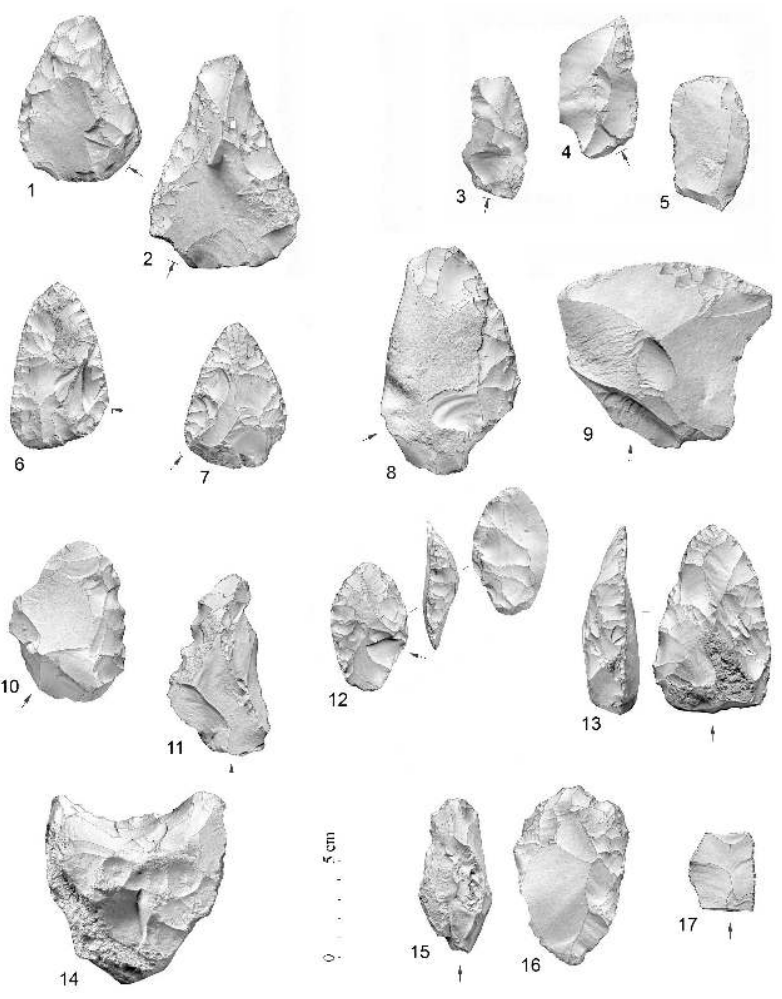

Figure 10 - Grand racloir utilisé pour percuter une matière dure organique, probablement dans le cadre d'une activité de boucherie. II porte en conséquence de grands et nombreux esquillements bifaciaux, principalement semi-circulaires et présentant une terminaison en escalier. La zone active, longue, est opposée à une zone préhensible constituée d'un plan de fracture de la dalle d'origine, formant un véritable dos.

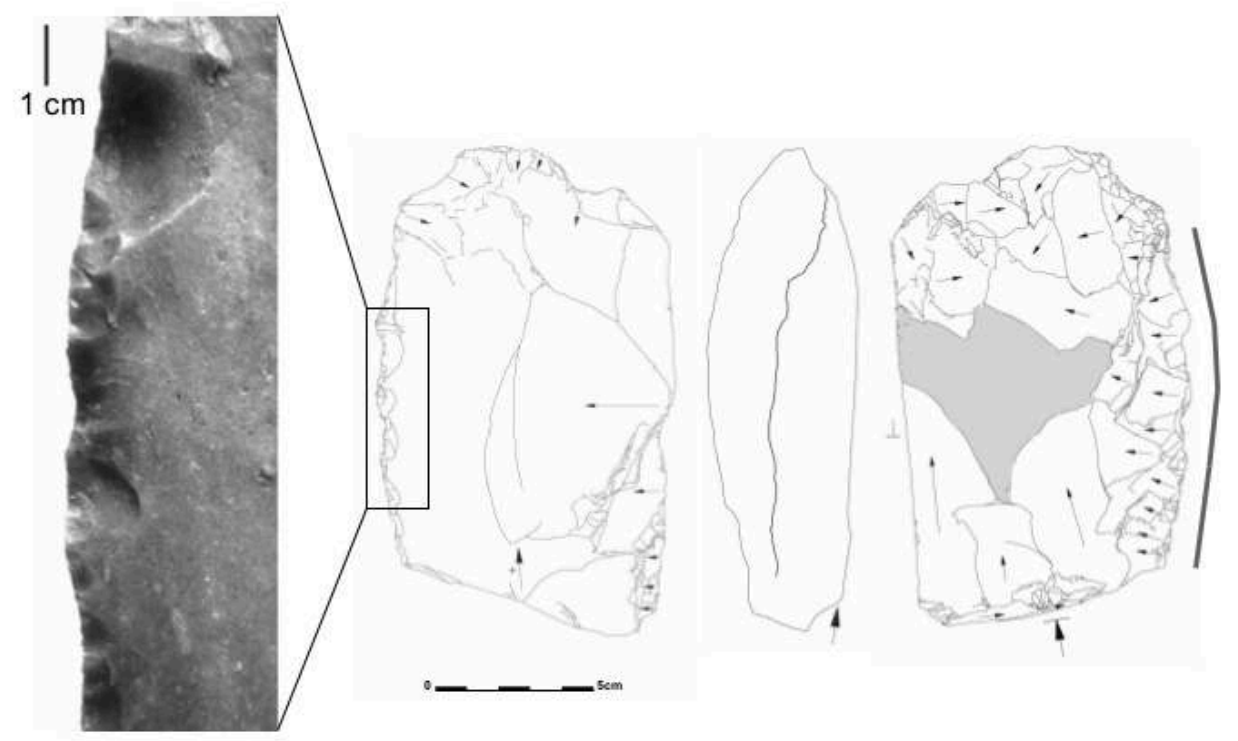




\section{Conclusion}

Le gisement de plein air de La Grande Vallée se situe dans une position et un contexte géologique tout à fait singulier. Au niveau d'un replat structural, l'unité 5 contenant les niveaux archéologiques a été préservée grâce à d'importants dépôts de versant qui sont véritablement venus sceller l'ensemble. Après les occupations par les Acheuléens, les niveaux archéologiques ont subi une mobilisation par solifluxion. Ce phénomène a modifié la répartition spatiale originelle des vestiges abandonnés par les hommes sans interférence stratigraphique. Sur la base des observations géologiques, les dépôts de l'unité 5 peuvent avoir été mis en place entre 350 et $600 \mathrm{ka}$, soit dans le second tiers du Pléistocène moyen. Cette estimation est confirmée par les dates préliminaires par thermoluminescence qui tendent à placer les niveaux $5 \mathrm{a}$ et $5 \mathrm{c}$ entre 400 et $500 \mathrm{ka}$. Il est probable que les datations en cours des niveaux inférieurs (5e, $5 \mathrm{~g}$ et $5 \mathrm{i}$ ) se situeront dans la même fourchette chronologique.

Bien que la faune n'ait pas été conservée, les études tracéologiques ainsi que la présence de nombreux silex brûlés montrent que le gisement n'est pas un simple atelier mais qu'il a été le lieu d'autres activités de subsistance. Le gisement de La Grande Vallée possède une industrie lithique diversifiée et une large variété typologique qui correspond à des concepts morpho-fonctionnels relativement bien définis et stabilisés, ainsi qu'à des chaînes opératoires clairement exprimées et réitérées. La qualité de cette industrie, à bien des points de vue, implique l'existence d'un long processus évolutif antérieur. L'industrie de La Grande Vallée confirme également que la capacité d'accommodation des groupes acheuléens à la variabilité de la matière première fut un aspect remarquable de leur comportement : accommodation aux galets des régions méridionales, aux rognons de silex étroits et allongés des régions septentrionales, et aux grandes dalles de silex du Turonien supérieur à La Grande Vallée, comme dans l'ensemble de la région du Seuil du Poitou et du sud de la Touraine.

Ce tour d'horizon des résultats préliminaires issus de cette première triennale de fouille permet de cerner d'emblée l'intérêt que revêt ce gisement dans la connaissance des premiers peuplements européens et en particulier dans la définition de ces cultures anciennes. En effet, en dépit de plus d'un siècle et demi de recherches, l'Acheuléen et son évolution restent encore délicats à cerner, comme l'a récemment souligné $\mathrm{E}$. Nicoud (2011) et antérieurement de nombreux auteurs dont A. Tuffreau (2004) parmi d'autres. Dans ce contexte, toute nouvelle découverte de cette lointaine période est fondamentale, venant enrichir un corpus restreint de gisements et permettant d'apporter de nouveaux éléments capitaux pour discuter de la nature et de la signification de ces assemblages très anciens.

\section{BIBLIOGRAPHIE}

AIRVAUX J. 1983 - Les industries acheuléennes de la région de Jarnac, Charente, I. Les hachereaux. Bulletin de la Société Préhistorique Française, 80, 2, p. 47-56. 
ANDERSON-GERFAUD P. 1981 - Contribution méthodologique à l'analyse des microtraces d'utilisation sur les outils préhistoriques. Bordeaux : Université de Bordeaux I, 1981. 314 p., Thèse de 3ème cycle.

BENN D.I. 1994 - Fabric shape and the interpretation of sedimentary fabric data. Journal of Sedimentary Research, A64, 4, p. 910-915.

BERTRAN P., FRANCOU B. et TEXIER J.-P. 1995 - Stratified Slope Deposits : the Stone-banked Sheets and Lobes Model. In : Slaymaker O. (Ed.), Steepland Geomorphology. London : Wiley \& Sons, p. 147-169.

BERTRAN P., HÉTU B., TEXIER J.-P., VAN STEIJN H. 1997 - Fabric characteristics of subaerial slope deposits. Sedimentology, 44, p. 1-16.

BERTRAN P. et TEXIER J.-P. 1999 - Facies and microfacies of slope deposits. Catena, 35, p. 99-121. BOURGUEIL B., CARIOU E., MOREAU P. 1976 - Carte Géologique au 1/50 000 de Vouneuil-surVienne, BRGM.

BRENET M. 2011 - Variabilité et signification des productions lithiques au Paléolithique moyen ancien. L'exemple de trois gisements de plein-air du Bergeracois (Dordogne, France). Bordeaux : Université de Bordeaux 1, $482 \mathrm{p}$. Thèse de Doctorat.

CARBONELL E., MOSQUERA M., OllÉ A., RODRÍGUEZ X.P., SAHNOUNI M., SALA R., VERGÈS J.M. 2001 - Structure morphotechnique de l'industrie lithique du Pléistocène inférieur et moyen $\mathrm{d}$ 'Atapuerca (Burgos, Espagne). L'Anthropologie, 105, p. 259-280.

CLAUD É. 2008 - Le statut fonctionnel des bifaces au Paléolithique moyen récent dans le Sud-Ouest de la France. Étude tracéologique intégrée des outillages des sites de La Graulet, La Conne de Bergerac, Combe Brune 2, Fonseigner et Chez-Pinaud/Jonzac. Bordeaux : Université de Bordeaux 1, 546 p. Thèse de Doctorat.

CLAUD É., BRENET M., MAURY S. et MOURRE V. 2009 - Étude expérimentale des macro-traces d'utilisation sur les tranchants des bifaces : caractérisation et potentiel diagnostique. Les Nouvelles de l'Archéologie, 118, p. 55-60.

CLAUD É., MOURRE V., THIÉBAUT C. et BRENET M. 2010 - Le recyclage au Paléolithique moyen. Des bifaces et des nucléus utilisés comme percuteurs. Archéopages, 29, p. 6-15.

DESPRIÉE J., VOINCHET P., TISSOUX H., MONCEL M.-H., ARZARELLO M., ROBIN S., BAHAIN J.-J., FALGUÈRES C., COURCIMAULT G., DÉPONT J., GAGEONNET R., MARQUER L., MESSAGER E., ABDESSADOK S., PUAUD S. 2010 - Lower and middle Pleistocene human settlements in the Middle Loire River Basin, Centre Region, France. Quaternary International, 223-224, p. 345-359.

FRITSCH R. 1972 - La station acheuléenne des Terriers près de La Revaudière (commune d'Yzeures-sur-Creuse, Indre-et-Loire). Revue Archéologique du Centre, $\mathrm{n}^{\circ}$ spécial Colloque d'Argenton, p. 8-14.

GERMOND G. 1982 - Une station acheuléenne au bord de la Dive du nord dans les Deux-Sèvres. Bulletin de la Société Préhistorique Française, 79, 10-12, p. 291-304.

GRATIER M. et MACAIRE J.-J. 1981 - Les alluvions anciennes de la Creuse à Yzeures-sur-Creuse (Indre-et-Loire). Paléoenvironnement et préhistoire. Gallia Préhistoire, 24, 1, p. 229-238.

GUILLIEN Y. 1941 - Les sablières de Jarnac. Bulletin de la société archéologique et historique de la Charente.

HALLEGOUËT B., HINGUANT S., GEBHARDT A., MONNIER J.-L. 1992 - Le gisement Paléolithique inférieur de Ménez-Drégan 1 (Plouhinec, Finistère). Bulletin de la Société préhistorique française, 89, 3, p. 77-81. 
HARRIS C., DAVIES M.C.R. et COUTARD J.-P. 1997 - Rates and processes of periglacial solifluction : an experimental approaches. Earth Surface Processes and Landforms, 22, p. 849-868.

JAMAGNE M. 2008 - Luvisols. In : Baize D. et Girard M.-C., Référentiel pédologique. Quae éd. : p. 223-232.

KEELEY L.H. 1977 - The functions of Palaeolithic flint tools. Scientific American, 237, p. 108-126.

KEELEY L.H. 1980 - Experimental Determination of Stone Tool Uses. Chicago : The University of Chicago Press, $212 \mathrm{p}$.

KEELEY L.H. 1993 - The utilization of lithic artifacts. Microwear analysis of Lithics. In :Singer R., Gladfelter B.-G. and Wymer J.-J. (Eds.), The lower Paleolithic site at Hoxne, England. Chicago : The University of Chicago Press, p. 129-138.

LENOBLE A. 2005 - Ruissellement et formation des sites préhistoriques : référentiel actualiste et exemples d'application au fossile. Oxford : British Archaeological Report International Series, $\mathrm{n}^{\circ} 1363,212 \mathrm{p}$.

LENOBLE A., BERTRAN P., BOULOGNE S., MASSON B. et VALLIN L. 2009 - Evolutions des niveaux archéologiques en contexte périglaciaire : apport de l'expérience Gavarnie. Les Nouvelles de l'Archéologie, 119, p. 16-20.

LENOBLE A., BERTRAN P. et LACRAMPE-CUYAUBÈRE F. 2007 - Solifluction-induced modifications of archaeological levels : simulation based on experimental data from a modern periglacial slope and application to French Palaeolithic sites. Journal of Archaeological Science, 35, p. 99-110.

LHOMME V. 2007 - Tools, space and behaviour in the Lower Palaeolithic : discoveries at Soucy in the Paris basin. Antiquity, 81, p. 536-554.

MITCHELL J.-C. 1998 - A use-wear analysis of selected british lower Paleolithic Handaxes with special reference to the site of Boxgrove (West Sussex). A study incorporating optical microscopy, computer aided image analysis and experimental archaeology. Oxford : Somerville College, 1998. 604p. Dissertation submitted for the degree of Doctor of Philosophy.

MURTON J. et FRENCH H.M. 1994 - Cryostructures in permafrost, Tuktoyaktuk coastlands, western arctic Canada. Canadian Journal of Earth Science, 31, p. 737-747.

NICOUD É. 2011 - Le phénomène acheuléen en Europe occidentale : Approche chronologique, technologie lithique et implications culturelles. Aix : Univ. Aix-en-Provence, 2011. 483 p. Thèse de Doctorat.

PARFITT S.A., Ashton N.M., LEWIS S.G., ABEL R.L., RUSSELL COOPE G., FIELD M.H, GALE R., HOARE P.G., LARKIN N.R., LEWIS M.D., KARLOUKOVSKI V., MAHER B.A., PEGLAR S.M., PREECE R.C., WHITTAKER J.E., STRINGER C.B. 2010 - Early Pleistocene human occupation the edge of the boreal zone in northwest Europe. Nature, 466, p. 229-233.

PATTE E. 1941 - Le Paléolithique dans le Centre-Ouest de La France. Paris : Masson, 207 p.

PATTE E. 1956 - Remarques sur les excursions du congrès préhistorique de 1956. Congrès préhistorique de France. Compte rendu de la XVe session, Poitiers-Angoulême 15-22 juillet, 1956, Société Préhistorique Française.

PATTE E. 1972 - Les alluvions de la Charente de Saint-Amand-de-Graves à Saint-Même. Mémoire de la société archéologique et historique de la Charente, p. 167-191.

PIPERNO M. (Ed.) 1999 - Notarchirico, un sito del Pleistocene medio antico nel bacino di Venosa. Venosa : Osanna, $621 \mathrm{p}$. 
RICHTER D., MOSER J., NAMI M., EIWANGER J. et MIKDAD A. 2010 - New chronometric data from Ifri n'Ammar (Morocco) and the chronostratigraphy of the Middle Palaeolithic in the Western Maghreb. Journal of Human Evolution, 59, p. 672-679.

ROBERTS M.B., PARFITT S.A. et POPE M.I. 1997 - Boxgrove, West Sussex, rescue excavations of a lower Palaeolithic landsurface (Boxgrove project B, 1989-1991). Proceedings of the Prehistoric Society, 63, p. 303-358.

SANTONJA M., LÓPEZ N., PÉREZ-GONZÁLEZA. (Eds) 1980 - Ocupaciones achelenses en el valle del Jarama (Arganda, Madrid). Madrid : Arqueología y Paleoecología, 1, Diputación Provincial.

SCHWARCZ H.P. 1994 - Current challenges to ESR dating. Quaternary Science Reviews, 13, p. 601-605.

SEMENOV S.A. 1964 - Prehistoric technology; an experimental study of the oldest tools and artefacts from traces of manufacture and wear. London : Cory, Adams et Mackay, $211 \mathrm{p}$.

TEXIER J.-P., BERTRAN P., COUTARD J.-P., FRANCOU B., GABERT P., GUADELLI J.-L., OZOUF J.-C., PLISSON H., RAYNAL J.-P. et VIVENT D. 1998 - TRANSIT, an experimental archaeological program in periglacial Environment : problematic, methodology, first results. Geoarchaeology, 13, 5, p. 433-473.

THIÉBAUT C., CLAUD E., MOURRE V., CHACÓN G., DAULNY L., ASSELIN G., BRENET M. et PARAVEL B. 2010 - Nucléus et bifaces présentant des traces de percussion au Paléolithique moyen. Quelles fonctions et quelles implications techno-économiques? Palethnologie.

http://www.palethnologie.org/images/stories/2010/fr-FR/Varia-2010-Thiebaut-FR.pdf

TRINGHAMR., COOPER G., ODELL G., VOYTEKB. et WHITMAN A. 1974 - Experimentation in the Formation of Edge Damage. A New Approach to Lithic Analysis. Journal of Field Archaeology, 1, p. 171-196.

TUFFREAU A. 2004 - L'Acheuléen : de l'Homo Erectus à l'homme de Néandertal. Paris : La Maison des Roches, $122 \mathrm{p}$.

TUFFREAU A., LAMOTTE A., GOVAL E. 2008 - Les industries acheuléennes de la France septentrionale. L'Anthropologie, 112, p. 104-139.

VOINCHET P., DESPRIÉE J., TISSOUX H., FALGUÈRES C., BAHAIN J.-J., GAGEONNET R., DÉPONT J., DOLO J.-M. 2010 - ESR Chronology of alluvial deposits and first human settlements of the Middle Loire Basin (Region Centre, France). Quaternary Geochronology, 5, 2-3, p. 381-384.

WASHBURNA.L. 1979 - Geocryology : A survey of periglacial processes and environments. London : Edward Arnold, 406 p.

WENBAN-SMITHF., BRIDGLAND D. 2001 - Palaeolithic Archaeology at the Swan Valley Community School, Swanscombe, Kent. Proceedings of the Prehistoric Society, 67, p. 219-259.

WYMER J. 1964 - Excavations at Barnfield Pit, 1955-1960. In : Ovey C.-D. (Eds.), The Swanscombe Skull. A Survey of Research on a Pleistocene Site. London : Royal Anthropological Institute of Great Britain and Ireland, Occasional Paper, 20, p. 19-61.

\section{RÉSUMÉS}

Dans cet article, sont présentés les résultats préliminaires des trois premières années de fouille programmée sur le gisement de La Grande Vallée à Colombiers dans la Vienne. L'étude de la séquence stratigraphique explorée sur une puissance de trois mètres a mis en évidence la 
présence de cinq niveaux archéologiques attribuables au Paléolithique inférieur. Les résultats archéologiques et pédostratigraphiques ainsi que les datations préliminaires par thermoluminescence sur silex brûlés convergent vers un âge des industries lithiques entre 400 et $500 \mathrm{ka}$. Bien que dénué d'éléments fauniques, la très grande richesse du gisement est incontestable et apporte un nouvel éclairage sur la connaissance des peuplements dans la zone centre-occidentale de la France durant cette phase. Les assemblages lithiques montrent une large variété typologique avec des concepts morpho-fonctionnels d'outils relativement stabilisés, ainsi que des chaînes opératoires spécialisées et réitérées. Les rapports entre l'industrie du Poitou et celles des régions septentrionales et méridionales indiquent la grande capacité d'accommodation des groupes acheuléens à d'importantes variations dans la présentation et la nature des matières premières lithiques.

Preliminary results of the first three years of programmed excavation on « La Grande Vallée » at Colombiers in Vienne are presented in this paper. The study of the stratigraphic sequence investigated on three meters deep highlighted the presence of five archaeological levels attributable to Lower Palaeolithic. Archaeological and pedostratigraphic results as well as thermoluminescence on burnt flint converge on an age for lithic industries between 400 and 500 ky. Although deprived of faunal elements, the high wealth of the site is indisputable and gives a new perspective on the knowledge of the settlements in the center-westerner zone of France during this period. Lithic assemblages show a large typological variety with morpho-functional concepts of relatively stabilized tools, as well as specialized and repeated " chaînes opératoires ". Links between the industries of Poitou and those of northern and southern regions indicate the high capacity of accomodation of Acheulean groups to important variations in the presentation and the nature of lithic raw materials.

\section{INDEX}

Mots-clés : Acheuléen, Paléolithique inférieur, biface, Pléistocène moyen, stratigraphie, processus de formation, taphonomie, datations TL, tracéologie

Keywords : Acheulean, Lower Palaeolithic, handaxe, Middle Pleistocene, statigraphy, formation processes, taphonomy, TL datings, usewear analysis

\section{AUTEURS}

\section{DAVID HÉRISSON}

Laboratoire HALMA-IPEL, UMR8164 du CNRS ; UFR de Géographie et d'aménagement, Avenue Paul Langevin, Université des Sciences et Technologies de Lille 1, 59655 Villeneuve d'Ascq Cedex, France - davidherisson@yahoo.fr

\section{JEAN AIRVAUX}

Chercheur indépendant ; 76, route de Bouresse, Mazerolles, 86320, Lussac-Les-Châteaux, France airvaux.jean@wanadoo.fr

\section{ARNAUD LENOBLE}

Laboratoire PACEA, UMR5199 du CNRS ; Université Bordeaux 1, Bat. B18, avenue des Facultés, 33405 Talence, France - a.lenoble@pacea.u-bordeaux1.fr 


\section{DANIEL RICHTER}

Université de Bayreuth, LS Geomorphologie, 95447 Bayreuth, Allemagne - daniel.richter@unibayreuth.de

\section{ÉMILIE CLAUD}

Inrap GSO Aquitaine, 356 avenue Jean Jaurès, Centres d'activités Les Echoppes, 33600 Pessac, et Laboratoire PACEA, équipe PPP, UMR 5199, Université Bordeaux 1, avenue des Facultés, Bat B18, 33405 Talence Cedex, France - emilie.claud@inrap.fr

\section{JÉRÔME PRIMAULT}

Service régional de l'archéologie de Poitou-Charentes, 102 Grand'Rue, BP 553, 86020 Poitiers ; UMR 7041 ARSCAN/ANTET du CNRS, Université Paris-X Nanterre, France -

jerome.primault@culture.gouv.fr 